RUNNING head: Cortisol, PTSD and Brain Volume

Salivary Cortisol and Regional Brain Volumes among Veterans with and without Posttraumatic Stress Disorder

Kimberly A. Babson, $\mathrm{PhD}^{1}$ Steven H. Woodward, $\mathrm{PhD}^{1,2}$ Marie Schaer, $\mathrm{MD}, \mathrm{PhD}^{3}$ Sandra E. Sephton, $\mathrm{PhD}^{4}$ Danny G. Kaloupek, $\mathrm{PhD}^{5,6}$

${ }^{1}$ National Center for PTSD-Dissemination and Training Division, VA Palo Alto Health Care System, Palo Alto, California

${ }^{2}$ Department of Psychiatry and Behavioral Sciences, Stanford University, Stanford, California

${ }^{3}$ Department of Psychiatry, University of Geneva School of Medicine, Geneva, Switzerland ${ }^{4}$ Department of Psychological and Brain Sciences, University of Louisville, Louisville, Kentucky

${ }^{5}$ National Center for PTSD- Behavioral Science Division, VA Boston Healthcare System, Boston, Massachusetts

${ }^{6}$ Boston University School of Medicine, Boston, Massachusetts

Keywords: posttraumatic stress disorder; veterans; cortisol; regional brain volumes;

hippocampus; amygdala

Corresponding Author

Kimberly A. Babson, Ph.D

795 Willow Road

Menlo Park, CA 94025

Email: Kimberly.babson@va.gov

Word Count Abstract: 236

Word Count Article: 3437

Tables: 2

Figures: 2

Supplemental Information: 1 


\begin{abstract}
Background: Human studies have often found that brain regions rich in glucocorticoid receptors exhibit smaller volume in samples with past trauma and ongoing stress; however, relatively little research has addressed the hypothesis that such smaller volumes can be traced to elevated circulating glucocorticoid hormones (GCs). This issue takes on renewed interest in light of recent proposals to treat symptoms of stress disorders such as posttraumatic stress disorder (PTSD) with exogenous synthetic GCs. We sought to examine the relation of circulating GCs to brain macrostructure among veterans with and without PTSD.

Methods: Participants $(n=90)$ included combat Veterans with and without PTSD. Veterans completed self-report surveys, home-based cortisol samples, reactive cortisol samples over the course of 2 serial Trier Social Stress Tests, a low-dose dexamethasone suppression test, and structural MRI brain imagining over the course of 3-5 days.
\end{abstract}

Results: No associations were observed between any salivary cortisol index and the volumes of the hippocampus or amygdala. A negative association was observed between evening basal cortisol and both FreeSurfer global volume and BrainImage supratentorial tissue volume. This effect was moderated by PTSD. Also observed was a positive association between reactive cortisol and these same brain volumes.

Conclusions: Estimates of cortical but not hippocampal or amygdala volume were moderately associated with evening basal salivary cortisol and cortisol reactivity to a social stressor. Existing models relating GC receptor density, circulating cortisol levels, and regional brain volumes received little support. 


\section{Salivary Cortisol and Regional Brain Volumes among Veterans with and without Posttraumatic Stress Disorder}

The release of glucocorticoid hormones (GCs) in response to acute stress orchestrates an array of adaptive and regulatory functions $(1,2)$. Chronic GC elevations are associated with structural modifications in the brain, the consequences of which are still unclear. Within the hippocampus, chronic GC elevations were found to be associated with regression of CA3 pyramidal apical dendritic arbors, and even with cell death $(3,4)$. Such results led to pioneering studies which found smaller hippocampal volume in posttraumatic stress disorder (PTSD) $(5,6)$, findings which have been generally replicated (7-9). Studies in animals soon extended beyond the hippocampus, demonstrating that exogenous glucocorticoid administration or restraint stress resulted in similar retraction of the apical dendritic arbors in the anterior cingulate cortex (ACC) of rats $(10,11)$. In apparent convergence, human studies have consistently found ACC cortical volume to be smaller in persons with $\operatorname{PTSD}(7,12)$.

While human studies have often found that brain regions rich in glucocorticoid receptors (GRs) exhibit smaller volume in samples with past trauma and ongoing stress, relatively little attention has been paid to the original hypothesis that these smaller volumes can be traced to elevated circulating GCs. Data accumulated over the past two decades have not strongly supported this proposition, which has taken on renewed interest in light of recent proposals to treat symptoms of stress disorders such as PTSD and Major Depressive Disorder (MDD) with exogenous synthetic GCs $(13,14)$. Across the psychiatric conditions in which this relationship has been assessed, including PTSD, MDD, psychosis, and chronic pain, approximately half of published studies have found either no relationship between GC levels and regional brain volumes, or positive relationships (see Supplemental Material S1 for a table of studies). In fact, 
two of the three studies employing PTSD samples failed to support the original hypothesis. Lindauer and colleagues (15) found that morning salivary cortisol levels were positively correlated with the volume of the right hippocampus. Neylan (16) found no relationship between salivary cortisol and hippocampal volume, but a positive correlation between cortisol and levels of n-acetylaspartate (NAA), a marker of neuronal integrity, in left hippocampus.

A similar distribution of confirmatory and disconfirmatory results characterizes studies in healthy samples and those with relevant medical conditions. In a quasi-experimental study on patients with Cushing's syndrome, Starkman (17) found that hippocampal enlargement correlated with pharmacologically-driven reductions in urinary free cortisol, but not plasma cortisol. Knoops (18) studied 575 middle-aged persons diagnosed with arterial disease and found modest negative associations between hippocampal volume and evening salivary cortisol levels, but not with an aggregate waking estimate. Kremen (19) studied 189 monozygotic twin pairs and found that two aggregate measures of waking salivary cortisol samples (mean and AUC) were negatively correlated with the thickness of a number of prefrontal cortical parcels, but not with the surface areas of those same parcels, nor with the volume of the hippocampus. The latter failure could have derived from reliance on a FreeSurfer-based estimate of hippocampal volume, as these have been shown to account for only $50 \%$ of the variance of manually-delineated volumes (20).

We sought to address the relation of circulating GCs to brain macrostructure in a relatively large sample of combat veterans with chronic severe PTSD and combat controls free of PTSD, current or lifetime. We have already reported on a number of associations between PTSD and regional brain volumes in this sample $(12,21)$. Importantly, both smaller hippocampal volume and smaller ACC volume have been confirmed in the PTSD subsample compared to the 
trauma-exposed controls, though the former effect was moderated by lifetime alcohol use disorder. Using salivary cortisol, we considered basal AM and PM cortisol, cortisol suppression by dexamethasone (DEX), cortisol reactivity to a social stressor, and the habituation of cortisol reactivity to that stressor. Examination of the latter was motivated by the proposal of McEwen and Margarinos (22) that delayed habituation to repeated stressors could resolve the apparent incongruency between the depressed cortisol levels often observed in PTSD samples (23-25) and brain volumetric effects supposedly traceable to elevated GCs. We also brought to bear two sets of regional brain volume measurement, one based on manual delineation and a second based on FreeSurfer. The former offered optimal measurement of specific volumes-of-interest such as hippocampus and amygdala, while the latter offered optimal measurement of the cerebral cortex and is in wide use today. Our interest in whole-cortex coverage derived, in part, from suggestions that GRs are more widely distributed in the primate than the rodent brain (26-28). In view of the distribution of GRs to all of these regions, we hypothesized that all measures of circulating GCs, basal, reactive, non-habituation of reactive over repeat stressors, and super-suppression by lowdose DEX, would be associated with smaller hippocampus, amygdala, ACC, and global cortical volumes.

\section{Methods and Materials}

\section{Participants}

Participants were U.S. Military veterans (females $=5.6 \% ; \mathrm{M}_{\mathrm{age}}=48.11, \mathrm{SD}=9.36$ ) with $(\mathrm{n}=45)$ and without $(\mathrm{n}=45)$ PTSD. Individuals participated in a parent study examining regional brain volumes as a function of PTSD diagnostic status and lifetime alcohol use disorders (12). A brief overview of the methods are described below, a more detailed overview of the methods have been described elsewhere (12). Individuals were excluded based on evidence of 
current or past central nervous system disease, psychosis, alcohol or substance use disorders within the past 6-months, and known contraindications for MRI. All participants reported exposure to combat-related trauma during the Gulf War $(n=33)$ or Vietnam War $(n=57)$ meeting DSM-IV defined Criterion A for the diagnosis. A majority of the sample (45.6\%) also experienced a Criteria A trauma at age 17 years or younger. Participants with PTSD, compared to those without PTSD, had fewer years of education, were less likely to be employed, were more likely to be non-Caucasian, and were more likely to smoke. In addition, those with PTSD had higher rates of current and lifetime MDD and were more likely to be taking psychoactive medications, principally SSRIs, SNRIs, and GABA agonists (see Table 1).

\section{Psychodiagnostics}

Diagnoses were obtained using the Clinician-Administered PTSD Scale (CAPS)(29) and the Structured Clinical Interview for DSM-IV (SCID) (30). Additional measures of declarative memory were obtained which are not germane to this report (31).

\section{Home-based Salivary Cortisol Collection and Quantification}

Participants provided home-based saliva samples in the morning (8:00AM) and evening (7:00PM) on two consecutive days (basal cortisol). All saliva samples were obtained using prelabeled Salivettes (Sarstedt, Nümbrecht, Germany). Participants were instructed not to eat or drink (except water) for 90 minutes prior to sample collection, and not to smoke for 60 minutes prior to sample collection. Participants also self-administered a low-dose DEX test over a separate two day period. This test consisted of an 8:00 AM saliva sample, ingestion of a $0.5 \mathrm{mg}$ tablet of DEX at 10:00 PM, followed by a saliva sample, and an 8:00 AM sample the following morning. Salivettes were brought or express-mailed to the laboratory. Salivettes were spun at 3000 RPM to remove saliva from the swabs. Saliva samples were then pipetted into $4 \mathrm{ml}$ 
Wheaton vials and stored at $-70 \mathrm{C}$ degrees. Samples were batch shipped over dry ice to PsychoNeuroEndocrine Research Laboratory in Louisville, KY for analysis by radioimmunoassay. This assay utilized a luminescence-based variation of the standard ELISA. An enzyme was used to convert a substrate, linked to cortisol within the sample, into a reaction product that emitted photons of light instead of developing a visible color (as in ELISA). We used a luminescence plate reader rather than a colorimetric one. Similar to ELISA, we used standards and both high and low controls to create a standard curve and estimate the reliability of our assay. Standards are samples with known concentrations of cortisol. Controls are large volume samples that are assayed each time we ran an assay, and the results are used for reliability estimates across multiple assays. Assay sensitivity was $.007 \mathrm{ug} / \mathrm{dL}$. Average interassay coefficients of variation was $6.53 \%$ and intra-assay coefficients of variability was $1.98 \%$ using high control. DEX assay sensitivity was $.007 \mathrm{ug} / \mathrm{dL}$. Average interassay coefficients of variability was $7.38 \%$ and intra-assay coefficients of variability was $4.52 \%$ using high control.

\section{Laboratory Procedures}

After undergoing electrode hook-up for psychophysiological recording and a series of neuropsychological tests, participants completed the first of two standard Trier Social Stress Tests (TSST) (32). Four saliva samples were obtained during each TSST, after the 10-minute speech preparation phase, upon completion of the speech and serial subtraction stressors, 20minutes post-stressors, and 40-minutes post-stressors. Immediately after each TSST, participants underwent two brief auditory oddball tone-counting procedures and two measures of declarative memory. After completing these tasks, participants sat quietly and/or read magazines for 20 minutes. The second trial of the TSST was then administered, followed by the same oddball and memory tasks. 


\section{Brain Imaging}

All participants were scanned using one of two 1.5 T GE Signa systems (Milwaukee, WI) MRI scanners. Scans were completed at the Radiology Center of the VA Palo Alto Health Care System (Palo Alto, California) or the Brain Imaging Center of McLean Hospital (Belmont, Massachusetts). A three-dimensional volumetric pulse sequence (TR=35 msec, TE=6 msec, flip angle $=45^{\circ}$, field of view $=24 \mathrm{~cm}^{2}$, number of excitations $=1$, image matrix size $=256 \times 192$, slice thickness $=1.5 \mathrm{~mm}$ to $1.7 \mathrm{~mm}$ ) was used to acquire coronal images. A board-certified neuroradiologist, blind to diagnosis, examined all scans for gross structural abnormalities. No participants were excluded on this basis. Additional details have been reported by Woodward and colleagues (12).

FreeSurfer version 5.3.0 (http://surfer.nmr.mgh.harvard.edu/) was used to extract regional global cortical volume, and volumes of rostral and caudal ACC parcels, hippocampus, and amygdala (33). A second set of estimates of supratentorial, ACC, hippocampal, and amygdala volumes were obtained via manual delineation in BrainImage (BrainImage 5.x; A. L. Reiss, Stanford University, Stanford, CA) (34). BrainImage-based values represent the sum of gray and white matter within delineated volumes as cross-site reliability assessment did not support the reliability of segmented volumes (12). Subcortical volumes were residualized against supratentorial volume (available in both FreeSurfer and BrainImage environments).

\section{Missing Data}

The maximum available data were used for all analyses. Ninety participants were available for analyses of basal and reactive cortisol. Eleven participants were excluded from the low-dose DEX analyses either because no DEX was detected in the saliva sample provided after ingesting DEX or because the post-DEX AM sample value was greater than 3 SDs above the 
whole sample mean. The same exclusion criterion was applied to basal cortisol samples and to those obtained during the TSST. As for AM cortisol concentrations, if both samples were available their maximum was used under the assumption that the maximum, even under a consecutive day sampling regime, would better reflect the peak of the individual's basal cortisol. PM cortisol values represent the mean of the two samples.

\section{Statistics}

Analyses of variance (ANOVAs; SPSS) were used to examine basal (morning vs evening) salivary cortisol concentrations as a function of PTSD diagnosis. Linear mixed effects models (35) were used to examine cortisol reactivity to the TSST. T-tests were used to examine cortisol suppression to low-dose DEX as a function of PTSD. Pearson product-moment correlations were used to examine the relations between global and regional brain volumes and basal cortisol, cortisol reactivity, and cortisol suppression. We required, a priori, that findings relating regional brain volumes to indices of HPA axis function be coherent across BrainImageand FreeSurfer-based measurement regimes. Findings not meeting this criterion were not reported. As a result, these were yoked comparisons not requiring further Type I error control. Indices of basal and reactive cortisol and cortisol suppression were treated as independent constructs. When appropriate, linear regression was employed to test moderation by smoking status, alcohol, study site, and PTSD diagnosis. A lowered standard was adopted when reporting possible moderation by these potential confounders: nominally significant moderation was reported if evidenced by either volumetric regime. 


\section{Results}

\section{Basal Cortisol}

A repeated-measures ANOVA of basal salivary cortisol yielded a main effect of time, $(F=135.83, p=.00)$, and a diagnosis by time interaction, $(F=11.40, p=.001)$. AM cortisol concentrations $(M=0.33 \mathrm{ug} / \mathrm{dL}, S D=.14)$ were higher than PM cortisol concentrations $(M=0.11$ $\mathrm{ug} / \mathrm{dL}, S D=.007)$. The PTSD subsample exhibited a smaller AM/PM cortisol difference than controls ${ }^{1}$.

Neither AM nor PM cortisol concentrations were associated with co-estimated regional volumes of the ACC, hippocampus, or amygdala (see Table 2). PM ( $p=.04)$, but not AM ( $p=$ .61), cortisol concentrations were negatively associated with FreeSurfer-derived global cortical volume and BrainImage-derived supratentorial volume (AM: $p=.59$; PM: $p=.02$; see Table 2).

After accounting for PTSD, smoking status did not moderate the relation between PM cortisol concentrations and FreeSurfer-derived global cortical volume $(\beta=.07, p=.80)$, or BrainImage-derived supratentorial volume $(\beta=.07, p=.82)$. After accounting for smoking status, moderation by PTSD of the relation between PM cortisol concentrations and FreeSurfer-derived global cortical volume approached significance $(\beta=.54, p=.06)$ and was nominally significant for the association between PM cortisol and BrainImage-derived supratentorial volume $(\beta=.63$, $p=.03$ ). Decomposition of the latter interaction suggested that greater PM cortisol concentrations were associated with smaller supratentorial volume among those with $\operatorname{PTSD}(\beta=-.41, p=.03)$, but not controls $(\beta=.10, p=.50)$. Neither study site nor lifetime alcohol use disorder moderated the relationships between regional brain volumes and basal cortisol concentrations (all $p$ 's $>.05$ ).

\section{Insert Figure 1}

\section{Cortisol Reactivity to a Social Stressor}


As depicted in Figure 1, salivary cortisol concentrations during the serial TSSTs exhibited expected main effects of trial $(F=97.10, p<.001)$ and sample $(F=38.44, p<.001)$, the latter comprising significant linear $(\beta=.031, p<.001)$, quadratic $(\beta=-.04, p<.001)$ and cubic $(\beta=$ $-.03, p<.001)$ effects. A smaller increase in salivary cortisol concentration on trial 2 led to a trial by sample interaction $(F=16.15, p<.001)$, comprising both linear $(\beta=-.04, p<.001)$ and quadratic effects $(\beta=.03, p<.001)$. PTSD diagnosis was associated with a nearly significant main effect $(F=3.18, p=.07)$, an interaction of diagnosis by trial, $(F=4.43, p=.03)$, and an interaction of diagnosis by sample, $(F=4.13, p=.007)$, underlying which only the linear increase over samples differentiated the groups $(\beta=-.02, p=.01)$. That linear increase over samples was steeper in controls. The three-way interaction of diagnosis, trial and sample was not significant ${ }^{2}$.

The range over samples was calculated in order to examine associations between cortisol responses to the TSST with regional brain volumes in a manner that utilized all available data and did not presume the location of the peak response. Consistent with the linear mixed effects analysis above, cortisol range over TSST samples exhibited a main effect of trial $(F=35.60, p<$ $.001)$, and a diagnosis by trial interaction $(F=4.00, p=.04)$. In this case, the main effect of diagnosis was also significant $(F=7.25, p<.001)$. Table 2 presents correlations between cortisol ranges on TSST trials 1 and 2 and FreeSurfer and BrainImage-derived volumes. Neither hippocampal, amygdalar, nor ACC volume was consistently correlated with cortisol reactivity to a social stressor. Instead, both aggregate measures of global cortical volume, FreeSurfer-derived cortical volume and BrainImage-derived supratentorial volume, covaried with reactive cortisol concentrations. In this case, however, salivary cortisol responses were positively associated with both brain tissue volumes. 
In addition, TSST trial 1 range was nominally correlated with the difference between AM and PM basal cortisol concentrations $(r=.24 ; p=.02)$. Review of bivariate plots confirmed that none of the above associations was influenced by outliers.

Linear regression models demonstrated that neither smoking status, lifetime alcohol use disorder, study site, nor PTSD moderated relations between cortisol response to the TSST and FreeSurfer-derived global cortical and BrainImage-derived supratentorial volume (all $p$ 's $>.05)$.

\section{Insert Figure 2}

\section{Suppression of AM Cortisol by Low-Dose Dexamethasone}

Cortisol suppression to low-dose DEX was larger in controls $(M=.31, S D=.25)$ than in participants with PTSD $(M=.21, S D=.17 ; \mathrm{t}=2.16, p=.03)$, a finding ascribable to higher AM cortisol levels in the former group. Table 2 presents correlations between cortisol suppression by low-dose DEX and regional brain volumes. Overall, cortisol suppression by low-dose DEX did not covary with FreeSurfer or BrainImage-derived global or regional volumes. Adjustment via ANCOVA for study site, SSRI status, and gender did not affect these results.

\section{Discussion}

This study examined candidate associations between regional brain volumes and salivary cortisol concentrations, basal, stress-reactive, and DEX-suppressed, in a relatively large sample of combat veterans with and without chronic severe PTSD. Participants with PTSD, most of whom also met criteria for MDD, exhibited less reduction of PM cortisol than controls, replicating the findings of Young and Breslau (36). Small but consistent negative relationships were observed between PM basal salivary cortisol concentrations and both automated and manual measures of global cortical volume. These observations are broadly consistent with the "neural endangerment" proposition deriving from the findings in hippocampus and extending to 
other regions rich in GRs; however, PM salivary cortisol levels were low in an absolute sense and no associations with hippocampal, amygdala or ACC volume were identified. Those without PTSD demonstrated greater cortisol suppression to low-dose DEX compared to those with PTSD. This finding may have been influenced by our method of AM cortisol sampling. However, past work by Wessa and colleagues (37) using cortisol awakening response (not DEX suppression) also showed cortisol suppression among those with PTSD.

Surprisingly, small but consistent positive associations were observed between cortisol reactivity to a social stressor and global cortical volume, whether estimated via automated or manual methods. We are not aware of a prior study that has assessed this specific relationship; however, Pruessner (38) also reported a positive relationship between cortisol reactivity to the TSST and hippocampal volume in a small sample of young adults. Such findings may draw attention to the frequency with which blunted cortisol responses to the TSST are associated with mood disorder and chronic stress (e.g. 39, 40,41). Not only did cortisol reactivity to a social stressor fail to exhibit negative associations with regional brain volumes, habituation of cortisol reactivity from the first to the second administration of the TSST was also not associated with any measure of brain volume. In sum, this study found no evidence of enhanced GR-mediated negative feedback in PTSD and no evidence that such enhanced feedback might be neuroprotective as reflected by regional brain volumes.

Though opposite in sign, the observed associations between PM cortisol versus TSST reactivity and cerebral cortical volume may not be incompatible if both are understood as indices of cortisol lability. This is straightforward in the case of the TSST response. In the case of evening basal cortisol it requires interpreting the failure to achieve lower evening levels as indicative of HPA inflexibility or hysteresis. Like blunted cortisol reactivity, higher evening 
cortisol concentrations have been associated with diverse conditions indicative of, or conferring risk for, chronic stress, including low socio-economic status (42), work strain (43), reduced cognitive function and frailty in the elderly (44), all-cause mortality following coronary bypass surgery (45) and cancer diagnosis (46). In this study, cortisol range during a stressor (TSST trial 1) and cortisol range over the day were modestly positively correlated. Noting that the positive relationships between basal PM cortisol, cortisol reactivity, and cortical volume were all modest, the existence of more complicated relationships involving these variables and other unmeasured and relatively more powerful factors is suggested.

The above results should be interpreted in light of a number of important limitations. First, the current study was based on cross-sectional data and the results purely correlational. Additional research is needed to prospectively examine the associations between GC levels and changes in neuroanatomical structures among individuals with PTSD. Second, because they are based on an almost exclusively male combat veteran sample, the results may not generalize to female samples or to samples composed of persons with civilian single-incident traumas. Third, these results were based solely on spot salivary cortisol concentrations. Future work could benefit from inclusion of more time-integrating plasma and/or urinary cortisol measures. Fourth, the current study was comprised solely of trauma-exposed Veterans. Examining the same associations in non-exposed Veterans could be revealing. Fifth, future studies could employ oversampling to address additional moderators/mediators including sex, depression, body mass index, and exposure to childhood trauma. Finally, our estimate of the cortisol awakening response would have been improved by the use of the multiple-sample methods now prevalent. In particular, our failure to replicate findings of enhanced low-dose DST in PTSD should be considered in that light. 
In conclusion, estimates of supratentorial and cortical volume, but not hippocampal or amygdala volume, were moderately negatively associated with evening basal salivary cortisol and positively correlated with cortisol reactivity to a social stressor, a pattern of results contrary to that predicted by familiar models relating circulating GCs to brain structure. 


\section{Acknowledgments}

Major funding for this work was provided under a VA/DoD assistance agreement to Drs. Woodward and Kaloupek from the U.S. Army Medical Research and Materiel Command (DoD) administered through the Institute for Medical Research (VA). Additional funding support was provided by a VA Clinical Science Research and Development Career Development Award (IK2 CX001023; Babson) The views expressed in this article are those of the authors and do not necessarily reflect the position or policy of the Department of Veterans Affairs or the United States Government. The Authors have no financial involvement with organizations whose financial interest may be affected by the material in the manuscript or which might potentially bias it. Dr. Steven H. Woodward (National Center for PTSD-Dissemination \& Training Division, VA Palo Alto Health Care System and Department of Psychiatry and Behavioral Sciences, Stanford School of Medicine) and Dr. Kimberly A. Babson (National Center for PTSDDissemination \& Training Division, VA Palo Alto Health Care System) had full access to all the data in the study and take responsibility for the integrity of the data and accuracy of the data analysis. Author contributions were as follows: Drs. Woodward and Kaloupek obtained the grant which supported the current study and conducted all procedures. Dr. Schaer scored and interpreted all brain images. Dr. Sephton conducted the analysis of cortisol samples. Drs. Woodward, Babson, and Kaloupek developed the idea for the current project. Drs. Woodward and Babson conducted all data analyses and initial manuscript development. All authors were involved in the writing and editing of the manuscript. Important institutional and technical support was also provided by Dr. Allan L. Reiss (Stanford University), Dr. Chis Streeter (Boston University), and Dr. Perry Renshaw (University of Utah).

Data from the parent project has been used to examine the associations between PTSD and regional cortical volumes in this sample $(12,21)$; however, previous publications have not examined the relation between cortisol and global and regional cortical volumes among this sample. 


\section{Footnote}

1. Analyses were also run after controlling for study site, gender, and use of

pharmacotherapy (SSRIs). Results remained the same with a main effect for time $(F=5.66, p=$ $.02)$, and a diagnosis by time interaction, $(F=6.90, p=.01)$.

2. LME analyses were also run after controlling for study site, gender, and the use of SSRIs. Results remained the same. 


\section{Financial Disclosures}

Dr. Babson has consulted for Insys Therapeutics within the past year. All other authors report no biomedical financial interests or potential conflicts of interests. 


\section{References}

1. McEwen BS. Effects of adverse experiences for brain structure and function. Biol Psychiatry. 2000;48:721-731.

2. de Kloet ER, Joels M, Holsboer F. Stress and the brain: from adaptation to disease. Nat Rev Neurosci. 2005;6:463-475.

3. Sapolsky RM, Krey LC, McEwen BS. Glucocorticoid-sensitive hippocampal neurons are involved in terminating the adrenocortical stress response. Proc Natl Acad Sci U S A. 1984;81:6174-6177.

4. Uno H, Lohmiller L, Thieme C, Kemnitz JW, Engle MJ, Roecker EB, Farrell PM. Brain damage induced by prenatal exposure to dexamethasone in fetal rhesus macaques. I. Hippocampus. Brain Res Dev Brain Res. 1990;53:157-167.

5. Bremner JD, Vythilingam M, Vermetten E, Adil J, Khan S, Nazeer A, Afzal N, McGlashan T, Elzinga B, Anderson GM, Heninger G, Southwick SM, Charney DS. Cortisol response to a cognitive stress challenge in posttraumatic stress disorder (PTSD) related to childhood abuse. Psychoneuroendocrinology. 2003;28:733-750.

6. Bremner JD, Randall P, Scott TM, Bronen RA, Seibyl JP, Southwick SM, Delaney RC, McCarthy G, Charney DS, Innis RB. MRI-based measurement of hippocampal volume in patients with combat- related posttraumatic stress disorder. Am J Psychiatry. 1995;152:973-981.

7. O'Doherty DC, Chitty KM, Saddiqui S, Bennett MR, Lagopoulos J. A systematic review and metaanalysis of magnetic resonance imaging measurement of structural volumes in posttraumatic stress disorder. Psychiatry Res. 2015;232:1-33.

8. Nemeroff CB, Bremner JD, Foa EB, Mayberg HS, North CS, Stein MB. Posttraumatic stress disorder: a state-of-the-science review. J Psychiatr Res. 2006;40:1-21.

9. Karl A, Schaefer M, Malta LS, Dorfel D, Rohleder N, Werner A. A meta-analysis of structural brain abnormalities in PTSD. Neurosci Biobehav Rev. 2006;30:1004-1031.

10. Cook SC, Wellman CL. Chronic stress alters dendritic morphology in rat medial prefrontal cortex. J Neurobiol. 2004;60:236-248.

11. Cerqueira JJ, Catania C, Sotiropoulos I, Schubert M, Kalisch R, Almeida OF, Auer DP, Sousa N. Corticosteroid status influences the volume of the rat cingulate cortex - a magnetic resonance imaging study. Journal of Psychiatric Research. 2005;39:451-460.

12. Woodward SH, Kaloupek DG, Streeter CC, Kimble MO, Reiss AL, Eliez S, Wald LL, Renshaw PF, Frederick BB, Lane B, Sheikh JI, Stegman WK, Kutter CJ, Stewart LP, Prestel RS, Arsenault NJ.

Hippocampal volume, PTSD, and alcoholism in combat veterans. Am J Psychiatry. 2006;163:674-681.

13. Wingenfeld $\mathrm{K}$, Wolf OT. Effects of cortisol on cognition in major depressive disorder, posttraumatic stress disorder and borderline personality disorder - 2014 Curt Richter Award Winner.

Psychoneuroendocrinology. 2015;51:282-295.

14. Yehuda R, Flory JD, Bierer LM, Henn-Haase C, Lehrner A, Desarnaud F, Makotkine I, Daskalakis NP, Marmar CR, Meaney MJ. Lower methylation of glucocorticoid receptor gene promoter 1F in peripheral blood of veterans with posttraumatic stress disorder. Biol Psychiatry. 2015;77:356-364.

15. Lindauer RJ, Olff M, van Meijel EP, Carlier IV, Gersons BP. Cortisol, learning, memory, and attention in relation to smaller hippocampal volume in police officers with posttraumatic stress disorder. Biol Psychiatry. 2006;59:171-177.

16. Neylan TC, Schuff N, Lenoci M, Yehuda R, Weiner MW, Marmar CR. Cortisol levels are positively correlated with hippocampal N-acetylaspartate. Biol Psychiatry. 2003;54:1118-1121.

17. Starkman MN, Giordani B, Gebarski SS, Berent S, Schork MA, Schteingart DE. Decrease in cortisol reverses human hippocampal atrophy following treatment of Cushing's disease. Biol Psychiatry. 1999;46:1595-1602. 
18. Knoops AJ, Gerritsen L, van der Graaf Y, Mali WP, Geerlings MI. Basal hypothalamic pituitary adrenal axis activity and hippocampal volumes: the SMART-Medea study. Biol Psychiatry. 2010;67:1191-1198. 19. Kremen WS, O'Brien RC, Panizzon MS, Prom-Wormley E, Eaves LJ, Eisen SA, Eyler LT, Hauger RL, Fennema-Notestine C, Fischl B, Grant MD, Hellhammer DH, Jak AJ, Jacobson KC, Jernigan TL, Lupien SJ, Lyons MJ, Mendoza SP, Neale MC, Seidman L, Thermenos HW, Tsuang MT, Dale AM, Franz CE. Salivary cortisol and prefrontal cortical thickness in middle-aged men: A twin study. Neuroimage. 2010;53:10931102.

20. Morey RA, Selgrade ES, Wagner HR, 2nd, Huettel SA, Wang L, McCarthy G. Scan-rescan reliability of subcortical brain volumes derived from automated segmentation. Human Brain Mapping. 2010;31:17511762.

21. Woodward SH, Kaloupek DG, Streeter CC, Martinez C, Schaer M, Eliez S. Decreased anterior cingulate volume in combat-related PTSD. Biological Psychiatry. 2006;59:582-587.

22. McEwen BS, Magarinos AM. Stress and hippocampal plasticity: implications for the pathophysiology of affective disorders. Hum Psychopharmacol. 2001;16:S7-S19.

23. Yehuda R, Kahana B, Binder-Brynes K, Southwick SM, Mason JW, Giller EL. Low urinary cortisol excretion in Holocaust survivors with posttraumatic stress disorder. Am J Psychiatry. 1995;152:982-986.

24. Yehuda R, Teicher MH, Trestman RL, Levengood RA, Siever LJ. Cortisol regulation in posttraumatic stress disorder and major depression: a chronobiological analysis. Biol Psychiatry. 1996;40:79-88.

25. Yehuda R, Halligan SL, Grossman R, Golier JA, Wong C. The cortisol and glucocorticoid receptor response to low dose dexamethasone administration in aging combat veterans and holocaust survivors with and without posttraumatic stress disorder. Biol Psychiatry. 2002;52:393-403.

26. Patel PD, Lopez JF, Lyons DM, Burke S, Wallace M, Schatzberg AF. Glucocorticoid and mineralocorticoid receptor mRNA expression in squirrel monkey brain. J Psychiatr Res. 2000;34:383-392.

27. Patel PD, Katz M, Karssen AM, Lyons DM. Stress-induced changes in corticosteroid receptor expression in primate hippocampus and prefrontal cortex. Psychoneuroendocrinology. 2008;33:360367.

28. Sanchez MM, Young L, Plotsky PM, Insel TR. Distribution of corticosteroid receptors in the rhesus brain: relative absence of glucocorticoid receptors in the hippocampal formation. J Neurosci. 2000;20:4657-4668.

29. Blake DD, Weathers FW, Nagy LM, Kaloupek DG, et al. The development of a Clinician-Administered PTSD Scale. J Trauma Stress. 1995;8:75-90.

30. First MB, Spitzer RL, Gibbon M, Williams JBW: Structured Clinical Interview for the DSM-IV Axis-I Disorders. New York, Biometrics Research Department, New York State Psychiatric Institute:; 1995. 31. Woodward SH, Kaloupek DG, Grande L, Stegman WK, Kutter CJ, Leskin L, Prestel R, Schaer M, Reiss AL, Eliez S. Hippocampal volume and declarative memory function in combat-related PTSD. Journal of the International Neuropsychological Society. 2009:1-10.

32. Kirschbaum C, Pirke KM, Hellhammer DH. The 'Trier Social Stress Test'--a tool for investigating psychobiological stress responses in a laboratory setting. Neuropsychobiology. 1993;28:76-81.

33. Desikan RS, Segonne F, Fischl B, Quinn BT, Dickerson BC, Blacker D, Buckner RL, Dale AM, Maguire

RP, Hyman BT, Albert MS, Killiany RJ. An automated labeling system for subdividing the human cerebral cortex on MRI scans into gyral based regions of interest. Neuroimage. 2006;31:968-980.

34. Kates WR, Abrams MT, Kaufmann WE, Breiter SN, Reiss AL. Reliability and validity of MRI measurement of the amygdala and hippocampus in children with fragile $X$ syndrome. Psychiatry Res. 1997;75:31-48.

35. Pinheiro J BD, DebRoy S, Sarkar D, R Core Team: nlme: linear and nonlinear mixed effects models. R package version 3.1-117. 2014.

36. Young EA, Breslau N. Saliva cortisol in posttraumatic stress disorder: a community epidemiologic study. Biol Psychiatry. 2004;56:205-209. 
37. Wessa M, Rohleder N, Kirschbaum C, Flor H. Altered cortisol awakening response in posttraumatic stress disorder. Psychoneuroendocrinology. 2006;31:209-215.

38. Pruessner M, Pruessner JC, Hellhammer DH, Bruce Pike G, Lupien SJ. The associations among hippocampal volume, cortisol reactivity, and memory performance in healthy young men. Psychiatry Res. 2007;155:1-10.

39. Petrowski K, Wintermann GB, Schaarschmidt M, Bornstein SR, Kirschbaum C. Blunted salivary and plasma cortisol response in patients with panic disorder under psychosocial stress. International Journal of Psychophysiology. 2013.

40. Peckins MK, Susman EJ, Negriff S, Noll JG, Trickett PK. Cortisol profiles: A test for adaptive calibration of the stress response system in maltreated and nonmaltreated youth-CORRIGENDUM. Dev Psychopathol. 2016:1-2.

41. Melhem NM, Keilp JG, Porta G, Oquendo MA, Burke A, Stanley B, Cooper TB, Mann JJ, Brent DA. Blunted HPA Axis Activity in Suicide Attempters Compared to those at High Risk for Suicidal Behavior. Neuropsychopharmacology. 2016;41:1447-1456.

42. Bann D, Chen H, Bonell C, Glynn NW, Fielding RA, Manini T, King AC, Pahor M, Mihalko SL, Gill TM, Life Study i. Socioeconomic differences in the benefits of structured physical activity compared with health education on the prevention of major mobility disability in older adults: the LIFE study. J Epidemiol Community Health. 2016.

43. Rystedt LW, Cropley M, Devereux JJ, Michalianou G. The relationship between long-term job strain and morning and evening saliva cortisol secretion among white-collar workers. J Occup Health Psychol. 2008;13:105-113.

44. Johar H, Emeny RT, Bidlingmaier M, Reincke M, Thorand B, Peters A, Heier M, Ladwig KH. Blunted diurnal cortisol pattern is associated with frailty: a cross-sectional study of 745 participants aged 65 to 90 years. J Clin Endocrinol Metab. 2014;99:E464-468.

45. Ronaldson A, Kidd T, Poole L, Leigh E, Jahangiri M, Steptoe A. Diurnal Cortisol Rhythm Is Associated With Adverse Cardiac Events and Mortality in Coronary Artery Bypass Patients. J Clin Endocrinol Metab. 2015;100:3676-3682.

46. Schrepf A, Thaker PH, Goodheart MJ, Bender D, Slavich GM, Dahmoush L, Penedo F, DeGeest K, Mendez L, Lubaroff DM, Cole SW, Sood AK, Lutgendorf SK. Diurnal cortisol and survival in epithelial ovarian cancer. Psychoneuroendocrinology. 2015;53:256-267. 
Table 1. Demographic and Predictor Variables as a Function of PTSD Diagnostic Status

\begin{tabular}{|c|c|c|c|}
\hline Variable & $\begin{array}{c}\text { PTSD } \\
\text { M(SD) or } \mathbf{N}(\%) \\
\end{array}$ & $\begin{array}{c}\text { No PTSD } \\
\text { M(SD) or N }(\%) \\
\end{array}$ & $t$ or $X^{2}$ \\
\hline \multicolumn{4}{|l|}{ Demographics } \\
\hline Age & $49.11(87.96)$ & $47.11(10.56)$ & -1.01 \\
\hline Gender & $3(6.70 \%)$ & $2(4.40 \%)$ & 0.21 \\
\hline Years of Education & $14.40(1.57)$ & $15.38(2.01)$ & $2.57 *$ \\
\hline Currently Employed & $16(35.60 \%)$ & $37(82.20 \%)$ & $20.24 * * *$ \\
\hline Ethnicity & & & $8.18 * * *$ \\
\hline Caucasian & $27(60.00 \%)$ & $39(86.70 \%)$ & \\
\hline Non-Caucasian & $18(40.00 \%)$ & $6(13.30 \%)$ & \\
\hline \multicolumn{4}{|c|}{ Current Psychopathology } \\
\hline $\begin{array}{l}\text { Major Depressive } \\
\text { Disorder }\end{array}$ & $34(75.60 \%)$ & $1(2.230 \%)$ & $50.91 * * *$ \\
\hline Panic Disorder & $1(2.20 \%)$ & $0(0.00 \%)$ & 1.01 \\
\hline Social Anxiety Disorder & $2(4.90 \%)$ & $0(0.00 \%)$ & 2.25 \\
\hline \multicolumn{4}{|c|}{ Lifetime Psychopathology } \\
\hline $\begin{array}{l}\text { Major Depressive } \\
\text { Disorder }\end{array}$ & $39(86.70 \%)$ & $10(22.20 \%)$ & $37.68 * * *$ \\
\hline Alcohol Abuse Disorder & $21(46.70 \%)$ & $18(40.00 \%)$ & 0.41 \\
\hline \multicolumn{4}{|c|}{ Psychotropic Medications } \\
\hline Prescribed Medication & $31(68.90 \%)$ & $5(11.10 \%)$ & $31.30 * * *$ \\
\hline SSRI & $21(46.70 \%)$ & $2(4.40 \%)$ & $21.08 * * *$ \\
\hline GABA Agonist & $12(26.7 \%)$ & $1(2.20 \%)$ & $10.88 * *$ \\
\hline SNRI & $10(22.20 \%)$ & $0(0.00 \%)$ & $11.25 * *$ \\
\hline $\begin{array}{l}\text { Alpha Adrenergic } \\
\text { Blocker }\end{array}$ & $4(8.90 \%)$ & $0(0.00 \%)$ & $4.19 *$ \\
\hline Beta Adrenergic Blocker & $2(4.40 \%)$ & $1(2.20 \%)$ & 0.35 \\
\hline $\begin{array}{l}\text { Tricyclic } \\
\text { Antidepressants }\end{array}$ & $4(8.90 \%)$ & $1(2.20 \%)$ & 1.91 \\
\hline Steroid & $2(4.40 \%)$ & $1(2.20 \%)$ & 0.34 \\
\hline Benzodiazapine & $3(6.70 \%)$ & $0(0.00 \%)$ & 3.10 \\
\hline \multicolumn{4}{|l|}{ Predictor Variables } \\
\hline Smoker & $15(44.10 \%)$ & $5(11.40 \%)$ & $10.79 * *$ \\
\hline $\begin{array}{l}\text { PTSD Symptom } \\
\text { Severity }\end{array}$ & $74.29(18.03)$ & $8.45(11.16)$ & $-21.15 * * *$ \\
\hline
\end{tabular}

Note: Gender = number $(\%)$ female; Currently Employed = number $(\%)$ currently employed; $\mathrm{SSRI}=$ selective serotonin reuptake inhibitor; SNRI = serotonin and norepinephrine reuptake inhibitor; Smoker $=$ number $(\%)$ of smokers.

$*=p<.05 ; * *=p<.01 ; * * *=p<.001$. 
Table 2. Correlations Between Basal, Reactive, and Low-Dose DEX Salivary Cortisol Concentrations and Regional Brain Volumes.

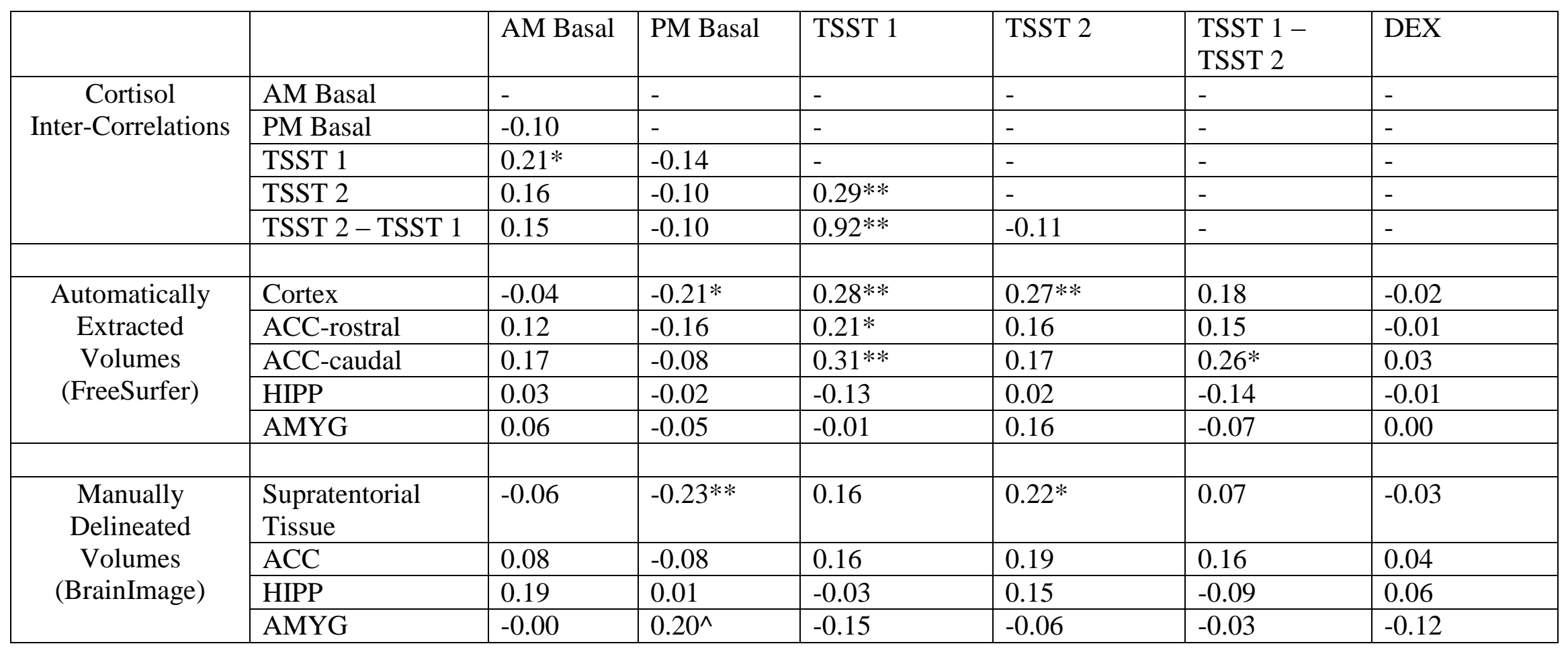

Note. $\mathrm{N}=90 ;$ AM Basal = Morning basal cortisol concentration; PM Basal = Evening basal cortisol concentration; TSST $1=$ Trier Social Stress Test trial 1 range; TSST 2 = Trier Social Stress Test trial 2 range; TSST 1 - TSST $2=$ Cortisol response persistence between trial 1 and 2 of the trier social stress test; DEX = cortisol concentration to low-dose dexamethasone; ACC = Anterior Cingulate Cortex; HIPP = Hippocampus; AMYG = Amygdala.

${ }^{\wedge} p=.05 ; * p<.05 ; * * p<.01 ; * * * p<.001$ (2-tailed tests). 


\section{Figure Legends}

Figure 1. Salivary cortisol concentration as a function of PTSD diagnostic status and trial during serial administration of the Trier Social Stress Test. Data represents mean cortisol concentration and standard error during each assessment phase. The timing of the assessments were as follows: $1=$ post speech preparation phase; $2=$ immediately post completion of the stressor; $3=20$ minutes post completion of the stressor; and $4=40$ minutes post completion of the stressor.

Figure 2. A scatterplot between Freesurfer-derived cortex volume estimates and salivary cortisol concentration over the course of trial 1 of the Trier Social Stress Test. This scatterplot demonstrates a lack of outliers within the data. 
Figure 1.

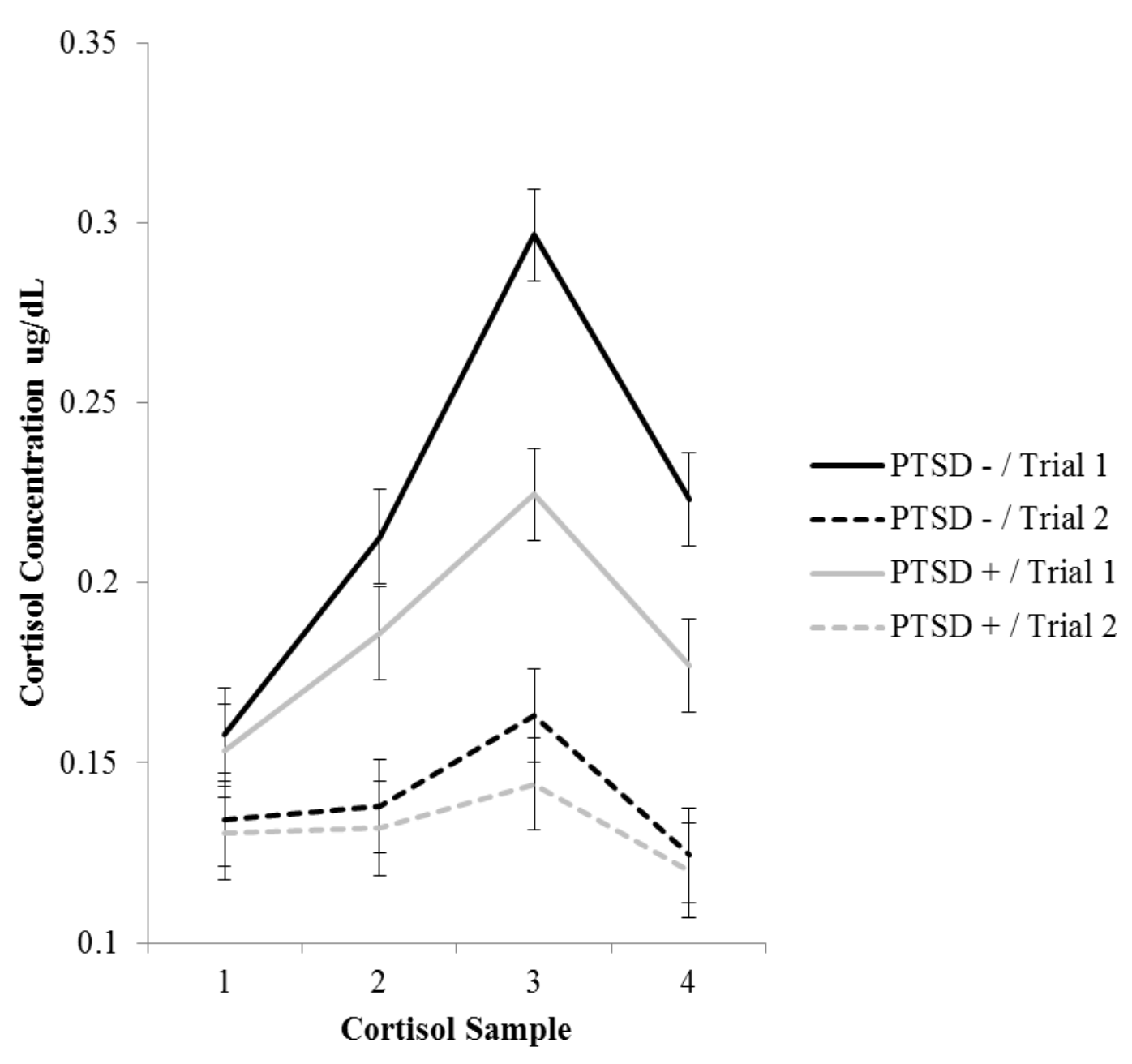

Figure 